\title{
Florence BERTHOLET, Anne BIELMAN SANCHEZ et Regula
} FREI-STOLBA (éd.), Egypte-Grèce-Rome. Les différents visages des femmes antiques

Travaux et colloques du séminaire d'épigraphie grecque et latine de l'IASA, 2002-2006, avant propos de Mireille CORBIER, Peter Lang, Bern 2008, 395 pages

\section{Violaine Sebillotte Cuchet}

\section{(2) OpenEdition} Journals

\section{Édition électronique}

URL : http://journals.openedition.org/clio/9499

DOI : 10.4000/clio.9499

ISSN : 1777-5299

\section{Éditeur}

Belin

Édition imprimée

Date de publication : 15 décembre 2009

Pagination : 257-261

ISSN : $1252-7017$

\section{Référence électronique}

Violaine Sebillotte Cuchet, «Florence berTholet, Anne bielman SANChez et Regula frei-Stolba (éd.), EgypteGrèce-Rome. Les différents visages des femmes antiques ", Clio. Histoire, femmes et sociétés [En ligne], 30 | 2009, mis en ligne le 01 février 2010, consulté le 21 septembre 2020. URL : http:// journals.openedition.org/clio/9499; DOI : https://doi.org/10.4000/clio.9499

Ce document a été généré automatiquement le 21 septembre 2020.

Tous droits réservés 


\section{Florence BERTHOLET, Anne BIELMAN SANCHEZ et Regula FREI-STOLBA (éd.), Egypte-Grèce-Rome. Les différents visages des femmes antiques}

Travaux et colloques du séminaire d'épigraphie grecque et latine de l'IASA, 2002-2006, avant propos de Mireille CORBIER, Peter Lang, Bern 2008, 395 pages

\section{Violaine Sebillotte Cuchet}

\section{RÉFÉRENCE}

Florence BERTHOLET, Anne BIELMAN SANCHEZ et Regula FREI-STOLBA (éd.), Egypte-Grèce-Rome. Les différents visages des femmes antiques. Travaux et colloques du séminaire d'épigraphie grecque et latine de l'IASA, 2002-2006, avant propos de Mireille CORBIER, Peter Lang, Bern 2008, 395 pages.

1 Cet ouvrage collectif issu de séminaires de l'Université de Lausanne présente des travaux portant sur le rapport des femmes au pouvoir dans trois aires linguistiques et culturelles de l'antiquité : l'Egypte pharaonique, le monde grec et le monde romain. La problématique d'ensemble rappelée dans l'avant-propos renvoie aux travaux de Fanny Cosandey sur la reine de France (La reine de France, symbole et pouvoir, Paris 2000). Il s'agit de souligner le caractère temporaire de l'autorité lorsqu'elle est exercée par une femme, laquelle ne saurait occuper le pouvoir que par délégation. Comme toute entreprise collective certaines contributions (publiées en français, allemand, anglais et italien) s'écartent de cette thématique ce qui justifie le titre très général choisi pour l'ouvrage. Les sources utilisées sont de natures diverses elles aussi, l'épigraphie fournissant la matière centrale très souvent adossée à des discussions de textes ou portant sur des dossiers iconographiques. 
2 Deux articles très riches et particulièrement cohérents traitent de l'Egypte pharaonique. Tous deux portent sur la même thématique, le pouvoir des reines. Le premier souligne l'absence de règle écrite codifiant le rôle royal, ce qui laisse, selon Annie Forgeau, une grande liberté d'action qui profite aux fortes personnalités. La pratique fait néanmoins de l'autorité monarchique un privilège masculin habituellement réservé au fils aîné. Le roi participe à la souveraineté par sa double nature, humaine par sa naissance, mais divine dans son essence puisqu'il est considéré comme héritant de la fonction de démiurge. La mère du pharaon a cependant un rôle primordial dans la définition sacrée de son fils. Dans l'iconographie, elle porte les attributs significatifs des substances supérieures : la dépouille de vautour, les sceptres ouas et ouadj, et le signe ankh. Dans les documents de l'ancien empire, le nom du roi est suivi de celui de sa mère, le matronyme restant prédominant pendant toute la période de construction de la monarchie. Par ailleurs, le double statut du pharaon, humain et divin, peut être partagé par son épouse car l'être royal se dédouble également en deux composantes, masculine et féminine. Même sans exercer l'autorité royale l'épouse est donc associée au caractère divin du pharaon. Elle porte le titre de «Celle qui voit Horus ", le dieu dynastique de Hiérakonpolis, archétype divin du roi vivant. La reine est la seule de toutes les femmes de la dynastie égyptienne et plus largement de tous les sujets, habilitée à "voir » le dieu, c'est-à-dire à dévoiler, lors du rituel du culte divin journalier, la statue cachée au cœur du sanctuaire. Durant le nouvel empire la reine devient le double théologique du monarque. Elle est donc susceptible d'assumer la totalité de la majesté royale. Le traitement stylistique des rois et des reines rend parfois l'identification sexuelle des souverains très difficile. On sait cependant que cinq reines, Nitocris, Neferou-Sebek, Hatchepsout, Merytaton, Taousert, assumèrent la totalité du pouvoir, ceci dans des circonstances de vacance d'héritier masculin. L'article suivant, en s'intéressant à la manière dont les anciens ont justifié le pouvoir des reines lagides à l'époque hellénistique, complète le précédent du point de vue de la thématique mais sans prendre la mesure, du point de vue du genre, des énoncés sur le caractère bisexué du pouvoir. Erhard Grzybek discute en fait très précisément l'idée qui se répand au début du III $^{\mathrm{e}}$ siècle av. J.-C., selon laquelle il est de longue tradition, en Egypte, que les femmes obtiennent la charge royale. L'argumentation de Grzybek est claire mais repose sur un postulat contestable : les seules reines connues de la tradition pharaonique ont tout fait pour passer pour des hommes (port de la barbe postiche, nom de trône choisi), un signe, dit-il, que les Egyptiens pensaient la royauté au masculin, ce qui signifie pour Grzybek qu'elle était réservée aux hommes. Et si, au $\mathrm{III}^{\mathrm{e}}$ siècle, l'idée se répand qu'il est normal qu'en Egypte les femmes gouvernent, c'est parce qu'à l'époque, une certaine Arsinoé a épousé son frère Ptolémée II et que celle-ci a orchestré la construction d'une tradition égyptienne susceptible de lui donner une dimension royale qui la situe à l'égal de son époux. Le raisonnement est très convaincant pour Arsinoé et l'écriture d'une première histoire de l'Egypte antique est problématique pour la période antérieure : l'indistinction sexuelle caractéristique des reines de l'époque pharaonique doit-elle être interprétée comme un signe de masculinisation ? Pour répondre à cette question il faudrait interroger la sexualisation des pharaons : les signes que nous disons masculins (barbe postiche, etc.) étaient-ils perçus comme tels par les Egyptiens? Seul un raisonnement appuyé sur les acquis théoriques de l'histoire du genre aurait permis de distinguer les signes de la royauté, une fonction sociale, et le sexe de celui ou celle qui la revêt. 
3 La partie sur le monde grec (Grèce propre et Asie mineure) comporte cinq articles explorant des domaines assez différents. Anne-Françoise Jaccottet propose une très intéressante interprétation du cratère de Derveni découvert en 1962 dans une riche tombe de Macédoine et daté du $\mathrm{IV}^{\mathrm{e}}$ siècle avant notre ère. En posant la question de la présence de cendres féminines mêlées à celles du défunt nommé par une inscription sur le vase, A.-F. Jaccottet propose de comprendre l'iconographie dionysiaque qui décore le vase comme le révélateur d'une manière particulière pour une femme d'accéder à une position héroïque, à l'égal d'un homme puisque sa sépulture comporte ici les caractéristiques traditionnelle de l'héroïsation. Marie Widmer s'attache ensuite à la figure de Laodice v qui épouse Antiochos III en 222 et est alors proclamée reine à Antioche. Plutôt que de la présenter comme une régente qui aurait régné en l'absence de son époux, M. Widmer la décrit comme une reine associée au pouvoir de son époux, un pouvoir dont les signes apparaissent dans les inscriptions, souvent des lettres de soutien dans lesquelles elle accorde des bienfaits aux populations des cités, mais également des décrets honorifiques. Ce travail rejoint les conclusions de la plupart des travaux actuels menés sur les reines hellénistiques qui interviennent autant dans le domaine dit privé que public. L'article suivant, de Jean-Yves Carrez-Maratray, sur la boucle de cheveux offerte par Bérénice II, épouse de Ptolémée III dans la seconde moitié $\mathrm{du} \mathrm{III}^{\mathrm{e}}$ siècle, évoque la question de la publicité faite autour des reines et s'insère assez mal, à mon avis, dans la problématique d'ensemble de l'ouvrage alors que le suivant, sur les reines au champ de bataille, est un utile inventaire des manières dont les reines agissent à la guerre, un thème jusqu'ici peu traité. Les reines y apparaissent recruteuses, commanditaires, stratèges, rarement combattantes. Malheureusement la conclusion tirée par Cédric Pillonel est très décevante : si elles ne combattent pas c'est qu'elles étaient plus "prudentes » que les rois! Anne Bielman Sanchez propose pour finir cette partie un long article sur l'iconographie funéraire des femmes de l'époque hellénistique et impériale ( $\mathrm{IV}^{\mathrm{e}}$ siècle avant notre ère - $\mathrm{II}^{\mathrm{e}}$ siècle de notre ère) dont elle ne manque pas de souligner le caractère extrêmement construit, par les hommes évidemment. Deux éléments frappent à la lecture : d'une part la manière dont les femmes ainsi honorées peuvent laisser leur propre nom et bénéficier à titre individuel d'un honneur public. Ceci est surtout vrai pour les prêtresses et est particulièrement net dans une série provenant de Smyrne et datée du IIème siècle avant J.-C. : les stèles marquées du sceau de la cité (une couronne dans laquelle s'inscrit le nom de l'honorant, «le peuple ») sont précisément des offrandes publiques. D'autre part les stèles qui honorent des prêtresses, des nourrices ou des femmes-médecins (les catégories les plus représentées), répartissent inégalement les signes valorisants. Selon A. Bielman, l'image reste le moyen le plus utilisé pour transmettre les mérites de la femme, bien plus que l'épitaphe, un constat qu'elle explique en recourant à un cliché de la littérature classique selon lequel la parure des femmes est le silence. Là aussi, on regrettera une conclusion aussi peu historicisée.

4 La dernière partie regroupe six articles consacrés à Rome. Le premier, de Barbara Scardigli, porte sur les figures des Vestales et des matrones sous la république romaine. Il est complété pour ainsi dire par celui de Nicole Boëls-Janssen sur les matrones de la fin de la république. L'idéal matronal de l'épouse fidèle que l'on peut lire dans les éloges funèbres, s'oppose, semble-t-il, au tableau des vies matrimoniales mouvementées caractéristiques de la fin de la république. Maria Grazia Granino Cecere propose ensuite une analyse des prêtresses du culte impérial dans l'Italie romaine. Restauratrices de monuments impériaux, elles peuvent ouvrir leurs domaines propres et leur richesse se 
mesure à la variété et la qualité de leurs dons. C'est à ce titre que Numisia se voit accorder le titre de "mère du municipe et de la colonie " par les citoyens de Teramo. L'article suivant est le premier et le seul à proposer une étude qui tienne explicitement compte des avancées effectuées dans le domaine du genre. Travaillant à partir de sources littéraires ne traitant que des élites sociales, Claude Emmanuelle Centlivres Challet montre que les caractéristiques féminines et masculines ne sont pas nécessairement opposées par les anciens et que les femmes ne constituent donc pas systématiquement une catégorie homogène et inférieure. Si l'idéal masculin est de devenir sénateur, l'idéal féminin une épouse de sénateur, les relations sociales au sein de la famille sont plus complexes puisqu'on peut louer une fille pour sa ressemblance avec son père et qu'une tante peut jouer le rôle du père décédé. C.E. Centlivres Challet décrit ainsi les femmes comme des témoins qui interagissent avec les hommes sans être confinées à un rôle ou une sphère. Doukaina G. Zanni analyse ensuite la figure de la Felicitas Temporum qui apparaît sur les monnaies émises sous les empereurs Nerva et Trajan. Figure de l'impératrice, la "félicité des temps » cristallise des vertus que l'on attribue également à l'empereur. Regula Frei-Stolba examine plus largement la figure de l'impératrice romaine qui s'inscrit dans une tradition de divinisation des épouses impériales depuis Livie.

5 Le livre propose des pistes de recherches et complète nombre dee thématiques ouvertes par l'histoire des femmes dans l'antiquité, notamment les spécialistes de l'époque hellénistique et impériale qui sont souvent également des spécialistes des inscriptions et de l'évergétisme. Il atteste de la vitalité de ce champ de recherches considérablement enrichi au cours des dernières années. Il atteste également de la difficulté, pour ces mêmes érudits, à intégrer les problématiques de genre dans une démarche qui se veut plus descriptive qu'explicative. La catégorie "femme " n'est jamais historicisée, sauf dans l'article de Challet dont l'analyse reste malheureusement à l'intérieur d'un corpus littéraire peu contextualisé. Ce grand écart entre une approche de genre confinée dans les représentations et une approche plus archéologique peu problématisée sur son objet, les femmes, explique les frustrations que peut ressentir le lecteur devant des conclusions finalement décevantes par rapport à l'importance de la matière mobilisée et à la finesse de la plupart des analyses. Un tel regret n'enlève rien au grand mérite de l'ouvrage: les antiquistes accumulent et diversifient les connaissances sur les sociétés antiques, ce qui est un acquis formidable. 